\title{
A Complication of Diaphragm Repair Using a Gore-Tex (Expanded Polytetrafluorethylene) Membrane: A Case Report
}

\author{
Seungwook Lee, M.D. ', Sung Yeon Hong, M.D. ${ }^{2}$, Jung A Son, M.D. ', Seungji Hyun, M.D. ', Seokjin Haam, M.D., Ph.D. ${ }^{1}$ \\ 'Department of Thoracic and Cardiovascular Surgery, Ajou University School of Medicine; ${ }^{2}$ Division of Hepatobiliary Surgery and Liver Transplantation, \\ Department of Surgery, Ajou University School of Medicine, Suwon, Korea
}

\section{ARTICLE INFO}

Received September 30, 2021

Revised November 16, 2021

Accepted November 16, 2021

Corresponding author

Seokjin Haam

Tel 82-31-219-4214

Fax 82-31-219-5215

E-mail haamsj@aumc.ac.kr

ORCID

https://orcid.org/0000-0002-0403-2216
A 65-year-old man underwent right trisectionectomy of the liver and reconstruction of the chest wall and diaphragm with a 2-mm Gore-Tex membrane due to recurrent hepatocellular carcinoma. After 3 years, the Gore-Tex membrane in the diaphragm migrated to the abdominal cavity and perforated the colon. We report a rare complication of a Gore-Tex membrane after diaphragm repair.

Keywords: Polytetrafluoroethylene, Foreign-body migration, Surgical wound infection, Intestinal perforation, Case report

\section{Case report}

The patient described in this report was a 65 -year-old man who had been diagnosed with hepatocellular carcinoma (HCC) 9 years prior. The patient had undergone 4 rounds of transarterial chemoembolization and radiotherapy (radiation dose, $600 \mathrm{cGy}$ ). Six years later, the patient had recurrent HCC and metastasis in the chest wall. He underwent right trisectionectomy of the liver and chest wall resection. At that time, the liver mass directly invaded the diaphragm; hence, en bloc resection of the liver mass and diaphragm was performed, as well as subsequent diaphragm reconstruction using a 2-mm Gore-Tex soft tissue membrane (W. L. Gore \& Associates, Flagstaff, AZ, USA) under laparotomy. Because the chest wall mass invaded the right seventh rib and the intercostal muscle, the rib and surrounding intercostal muscles were resected with a sufficient resection margin. Subsequent chest wall reconstruction was performed using another 2-mm Gore-Tex membrane. No postoperative complications or HCC recurrence was observed for 3 years. The patient visited the outpatient clinic once due to chest wall pain a year ago, but only conservative management was performed because no abnormal findings were observed on a physical examination or computed tomography (CT) (Fig. 1A).
One year later, the patient again visited the outpatient clinic complaining of chest wall pain and redness at the previous chest surgical site that had persisted for 1 month. On CT, a $10-\mathrm{cm}$ abscess was observed in the chest wall; hence, debridement and a local flap with right thigh skin were performed. The Gore-Tex membrane attached to the chest wall was clean, and no fistula connecting to the thoracic or abdominal cavity was found (Fig. 1B).

The patient returned to the hospital due to wound dehiscence after 6 months. No definite abscess was observed in the chest wall on CT scans; however, the Gore-Tex membrane that had been attached to the diaphragm had migrated into the abdominal cavity (Fig. 1C). Therefore, the treatment plan was to remove the migrated Gore-Tex membrane and the other membrane that had been placed on the chest wall. The chest wall was relatively clean with no visible infection or abscess, and the attached Gore-Tex membrane was easily removed. After performing laparotomy, we observed that the other Gore-Tex membrane inside the abdominal cavity was curled and that the end of the membrane was very sharp and hard, with this tip causing perforation of the ascending colon (Fig. 2). Because severe infection and adhesions were observed around the perforation site, right hemicolectomy, partial duodenectomy of the second portion, and gastrojejunostomy were performed. 

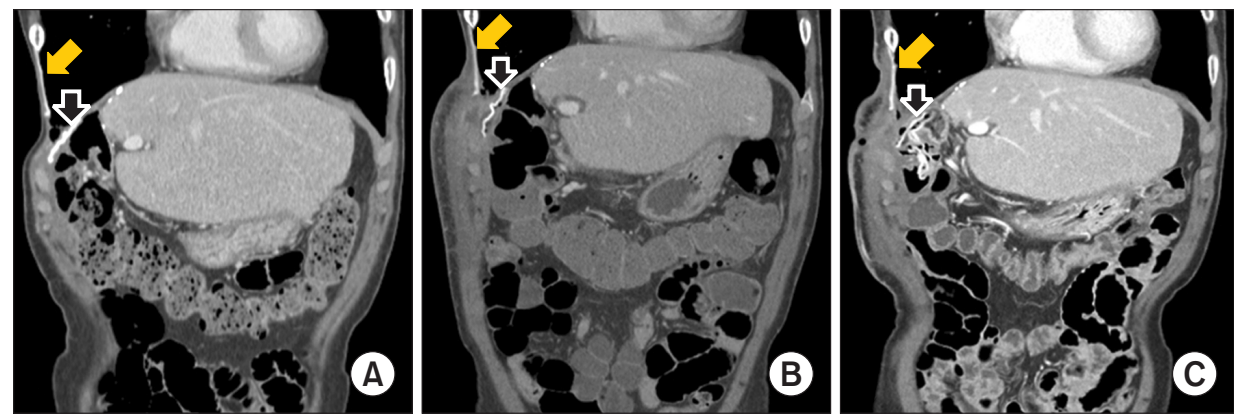

Fig. 1. Serial coronal views of abdominal computed tomographic scans when the patient was treated conservatively at an outpatient clinic 2 years after trisectionectomy (A), when he was treated with debridement and a skin flap 3 years after trisectionectomy (B), and before Gore-Tex removal surgery $(\mathrm{C})$. The solid yellow arrow shows the repaired chest wall with the Gore-Tex membrane and the empty white arrows point to the repaired diaphragm with Gore-Tex membrane.

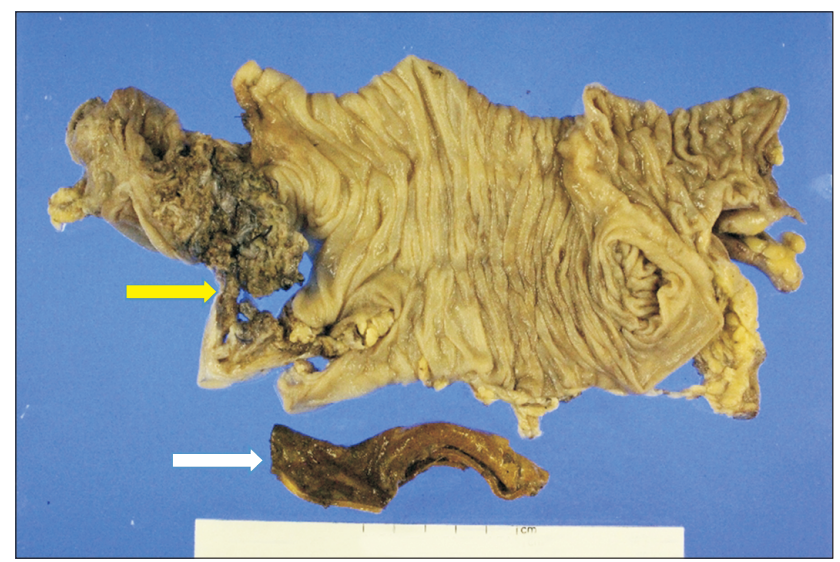

Fig. 2. Rolled Gore-Tex membrane (white arrow) and perforated ascending colon specimen (yellow arrow).

Due to the presence of severely fibrotic tissues between the thoracic cavity and the abdominal cavity, we determined that abdominal organ herniation would not occur. Therefore, no additional diaphragmatic repair was performed. The patient has been followed up through outpatient care for 6 months after surgery without any further complications (Fig. 3). The patient provided written informed consent for the publication of his clinical details and images.

\section{Discussion}

Although a small resection site in the diaphragm can be directly closed, a larger defect necessitates repair using a prosthesis. The Gore-Tex membrane is one of the most commonly used prostheses for diaphragm reconstruction. It is strong, flexible, and relatively inexpensive, and can easily be fitted to match the size of the resected area. However, the Gore-Tex membrane lacks elasticity; it could shrink and lead to unexpected hyaline necrosis with calci-

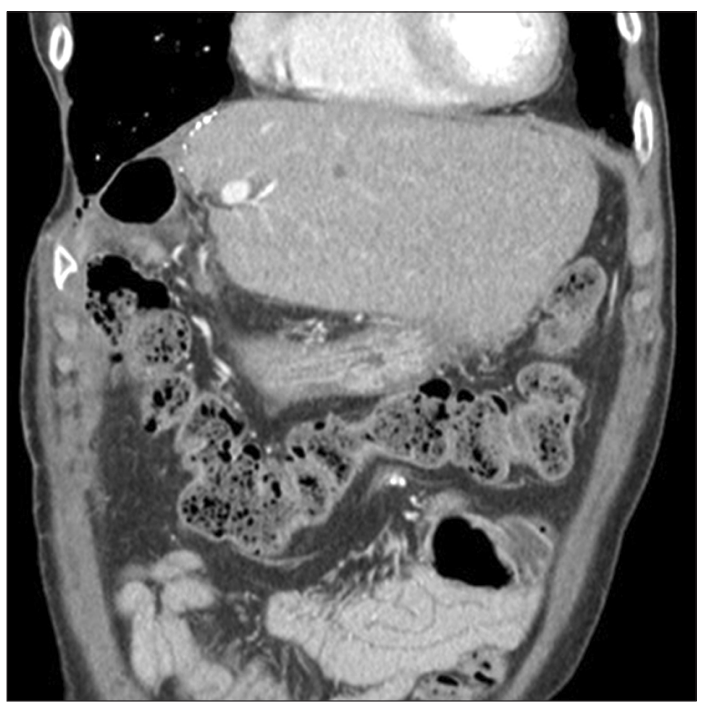

Fig. 3. Computed tomography scan 1 year after surgery.

fication [1,2]. Additionally, it has the drawback of being susceptible to infection.

Gore-Tex is also frequently used as an artificial vascular graft in vascular diseases, and Gore-Tex grafts used in vascular surgery have been reported to cause perforation in the adjacent organs $[2,3]$. However, to the best of our knowledge, there has been no reported case of perforation of an abdominal organ by migration of the Gore-Tex membrane used for diaphragm repair. In the case described in this report, chest wall and diaphragm reconstruction were performed using a Gore-Tex membrane after chest wall and diaphragm resection, and no specific abnormal findings were observed for 3 years. The patient visited our hospital due to a chest wall abscess 1 year ago-before the events described herein-and underwent surgical drainage and skin transposition. However, at that time, no fistula of the 
chest wound from the thoracic cavity was observed on CT scans or in the operating room. Moreover, the wound was clean, and no signs of infection were found. The results of wound and blood cultures for bacteria were negative. It is not clear why the Gore-Tex membrane moved into the abdominal cavity, but we hypothesize that the infection had not been completely controlled and that the infection and inflammation spread to the diaphragm, leading to weakness of the diaphragm tissue, detachment of the Gore-Tex membrane from the diaphragm, and migration into the abdominal cavity.

Despite colon perforation, the patient did not present any symptoms or signs of systemic infection, such as panperitonitis. The reason for this may have been that the chest wall infection had spread to the diaphragm membrane, causing inflammation and adhesion in the adjacent tissues, which in turn resulted in the isolation of the colon perforation site. Upon observation, we found severe fibrosis and adhesion only around the diaphragm and the perforation site, with the other areas remaining relatively clean.

Occasional reports have described infections of Gore-Tex membranes used for diaphragm or pericardium repairs; in most cases, the membrane needed to be removed for infection control $[4,5]$. Although definitive guidelines for the treatment of Gore-Tex infections do not exist, Kao et al. [6] suggested an algorithm for the management of prosthesis infections. Primarily, patients with clinical findings suggesting enterocutaneous/prosthesis fistula and active smokers should undergo prosthesis removal. Depending on their material, some prostheses can be maintained even if there is an infection, but it is recommended to remove Gore-Tex prostheses [6].

Membrane removal causes tissue defects, but another repair using an artificial material will greatly increase the risk of infection. In the present case, severe fibrosis occurred at the original membrane location, and repair using a prosthesis was not performed again. Potential complications such as abdominal organ herniation have not been observed as of 6 months after surgery.

In general, the Gore-Tex membrane is the most commonly applied material for diaphragm repair. Nevertheless, bovine pericardium, acellular porcine dermal collagen, or acellular human cadaveric dermis can also be used [7]. Among these materials, acellular dermal matrix reportedly acts as a tissue-conductive scaffold and is thus more physiologic and superior in terms of infection resistance [8].

In conclusion, a Gore-Tex membrane used for diaphragm repair could migrate into the abdominal cavity and cause perforation of an abdominal organ. Therefore, even if a patient has no symptoms, it may be necessary to check the location of the Gore-Tex membrane through periodic examinations; in particular, when signs of infection are observed, the possibility of Gore-Tex membrane migration should be considered.

\section{Conflict of interest}

No potential conflict of interest relevant to this article was reported.

\section{ORCID}

Seungwook Lee: https://orcid.org/0000-0003-1616-3595

Sung Yeon Hong: https://orcid.org/0000-0001-6993-9583

Jung A Son: https://orcid.org/0000-0002-0736-1226

Seungji Hyun: https://orcid.org/0000-0002-2578-3510

Seokjin Haam: https://orcid.org/0000-0002-0403-2216

\section{References}

1. Bertoglio P, Garelli E, Brandolini J, et al. Surgical management and reconstruction of diaphragm, pericardium and chest wall in mesothelioma surgery: a review. J Clin Med 2021;10:2330.

2. Weiss JP, Lorenzo FV, Campbell CD, Myerowitz RL, Webster MW. The behavior of infected arterial prostheses of expanded polytetrafluoroethylene (Gore-Tex). J Thorac Cardiovasc Surg 1977;73:630-6.

3. Woo HY, Hong SK, Cho JH, et al. Complications of polytetrafluoroethylene graft use in middle hepatic vein reconstruction in living donor liver transplantation: a retrospective, single-centre, long-term, real-world experience. Transpl Int 2021;34:455-64.

4. Avci T, Tasoglu I, Aygun E, Pac M. An unusual complication and imaging of PTFE membrane: report of a case. J Rare Dis Res Treat 2016;1:78-80.

5. Tatum RP, Shalhub S, Oelschlager BK, Pellegrini CA. Complications of PTFE mesh at the diaphragmatic hiatus. J Gastrointest Surg 2008; 12:953-7.

6. Kao AM, Arnold MR, Augenstein VA, Heniford BT. Prevention and treatment strategies for mesh infection in abdominal wall reconstruction. Plast Reconstr Surg 2018;142(3 Suppl):149S-155S.

7. Solli P, Bertolaccini L, Brandolini J, Pardolesi A. Reconstructive techniques after diaphragm resection and use of the diaphragmatic flap in thoracic surgery. Shanghai Chest 2017;1:21.

8. Bassuner JK, Rice DC, Antonoff MB, et al. Polytetrafluoroethylene or acellular dermal matrix for diaphragmatic reconstruction? Ann Thorac Surg 2017;103:1710-4. 